\title{
SPERNER PROPERTY AND FINITE-DIMENSIONAL GORENSTEIN ALGEBRAS ASSOCIATED TO MATROIDS
}

\author{
TOSHIAKI MAENO AND YASUHIDE NUMATA
}

\begin{abstract}
We prove the Lefschetz property for a certain class of finite-dimensional Gorenstein algebras associated to matroids. Our result implies the Sperner property of the vector space lattice. More generally, it is shown that the modular geometric lattice has the Sperner property. We also discuss the Gröbner fan of the defining ideal of our Gorenstein algebra.
\end{abstract}

Introduction. The Lefschetz property for Artinian Gorenstein rings is a ring-theoretic abstraction of the Hard Lefschetz theorem for compact Kähler manifolds. Stanley developed the ideas of applications of the Lefschetz property to combinatorial problems. For example, he showed in [18] the Sperner property of the Bruhat ordering on the Weyl groups based on the Hard Lefschetz theorem for the flag varieties. One of the main topics of the present paper is an application of the Lefschetz property for a certain kind of finite-dimensional Gorenstein algebras to the Sperner property of the vector space lattice $V(q, n)$ consisting of the linear subspaces of the vector space $\mathbb{F}_{q}^{n}$. A finite ranked poset $P=\bigcup_{i \geq 0} P_{i}$ with the level sets $P_{i}$ is said to have the Sperner property if the maximal cardinality of antichains of $P$ is equal to $\max _{i}\left(\# P_{i}\right)$.

For a given ranked poset $P=\bigcup_{i} P_{i}$, let $V_{i}$ be the vector space spanned by the elements of $P_{i}$. The Sperner property for $P$ can be shown by constructing a sequence $\left(f_{0}, f_{1}, f_{2}, \ldots\right)$ of linear maps $f_{i}$ : $V_{i} \rightarrow V_{i+1}$ satisfying a certain condition. Let $A^{(i)}=\left(a_{u v}^{(i)}\right)_{u \in P_{i}, v \in P_{i+1}}$

2010 AMS Mathematics subject classification. Primary 13E10, Secondary 05B35, 06A11, 13H10.

Keywords and phrases. Sperner property, Lefschetz property, matroid, Gorenstein algebra.

The first author is supported by Grant-in-Aid for Scientific Research.

Received by the editors on August 8, 2014, and in revised form on July 3, 2015. DOI: $10.1216 / \mathrm{JCA}-2016-8-4-549$ 
be the matrix representing $f_{i}$, i.e.,

$$
f_{i}(u)=\sum_{v \in P_{i+1}} a_{u v}^{(i)} v, \quad u \in P_{i} .
$$

If every matrix $A^{(i)}$ satisfies the condition $a_{u v}^{(i)} \neq 0 \Rightarrow u<v$ and is of full rank, then $P$ has the Sperner property (see, e.g., [10] for details).

The Sperner property of the vector space lattice $V(q, n)$ can be deduced from the result on the rank of its incidence matrices due to Kantor [11]. We will give another proof of the Sperner property of $V(q, n)$ by the construction of a finite-dimensional Gorenstein algebra $A_{M(q, n)}$ associated to the matroid $M(q, n)$ on the finite projective space $\mathbb{P}^{n-1}\left(\mathbb{F}_{q}\right)$ and by showing that $A_{M(q, n)}$ has the Lefschetz property.

Our construction can be done for general matroids. For a matroid $M$ and its bases $\mathcal{B}$, we introduce a polynomial $\Phi_{M}:=\sum_{B \in \mathcal{B}} x_{B}$. The Gorenstein algebra $A_{M}$ will be defined to be the quotient algebra of the ring of the differential polynomials by the annihilator Ann $\Phi_{M}$ of $\Phi_{M}$. We will generalize the results for the matroid $M(q, n)$ to the case of matroids corresponding to modular geometric lattices. The Sperner property of the modular geometric lattice has been proved by Baker [1]. Our argument based on the Gorenstein algebra $A_{M}$ leads us to another proof of Baker's result.

For a general polynomial $F$, though $F$ has all the information on the annihilator Ann $F$ in principle, the combinatorial structure of Ann $F$ is quite delicate in general, so it is difficult to describe directly from $F$. It is remarkable that in our case the Gröbner fan $G\left(\operatorname{Ann} \Phi_{M(q, n)}\right)$ of the annihilator of $\Phi_{M(q, n)}$ is a refinement of that of the principal ideal generated by $\Phi_{M(q, n)}$, which is also a consequence of our main theorem. As discussed in [2], the Gröbner fan of an ideal is often difficult to compute. We will see that $G\left(\operatorname{Ann} \Phi_{M(q, n)}\right)$ can be recovered from the tropical hypersurfaces of certain polynomials defined by the bases of the linear subspaces of $\mathbb{P}^{n-1}\left(\mathbb{F}_{q}\right)$.

The main results of this paper have been given in $[\mathbf{1 2}, \mathbf{1 3}]$.

\section{Finite-dimensional Gorenstein algebras and Lefschetz} property. In this section, we summarize some fundamental results on the structure of finite-dimensional Gorenstein algebras and on the Lefschetz property, which will be used in the subsequent sections. 
Definition 1.1. Let $A=\oplus_{d=0}^{D} A_{d}, A_{D} \neq 0$, be a graded Artinian algebra. We say that $A$ has the strong Lefschetz property (in the narrow sense) if there exists an element $L \in A_{1}$ such that the multiplication map

$$
\times L^{D-2 i}: A_{i} \longrightarrow A_{D-i}
$$

is bijective for $i=0, \ldots,[D / 2]$.

In the rest of this paper, we consider the Gorenstein algebras that are finite-dimensional over a field $k$ of characteristic zero.

Definition 1.2. (see [16, Chapter 5, 6.5]). A finite-dimensional graded $k$-algebra $A=\oplus_{d=0}^{D} A_{d}$ is called the Poincaré duality algebra if $\operatorname{dim}_{k} A_{D}=1$ and the bilinear pairing

$$
A_{d} \times A_{D-d} \longrightarrow A_{D} \cong k
$$

is non-degenerate for $d=0, \ldots,[D / 2]$.

The following is a well-known fact (see, e.g., $[\mathbf{6}, \mathbf{1 0}, \mathbf{1 4}]$ ).

Proposition 1.3. A graded Artinian k-algebra $A$ is a Poincaré duality algebra if and only if $A$ is Gorenstein.

Corollary 1.4. The tensor product of two graded Artinian Gorenstein $k$-algebras is again Gorenstein.

Let $P=k\left[x_{1}, \ldots, x_{n}\right]$ and $Q=k\left[X_{1}, \ldots, X_{n}\right]$ be polynomial rings over $k$. We may regard $P$ as a $Q$-module via the identification $X_{i}=\partial / \partial x_{i}, i=1, \ldots, n$. For a polynomial $F(x) \in P$, denote by Ann $F$ the ideal of $Q$ generated by the differential polynomials annihilating $F$, i.e.,

$$
\text { Ann } F:=\{\varphi(X) \in Q \mid \varphi(X) F(x)=0\} .
$$

The following is immediate from the theory of inverse systems (see $[3,5,7])$.

Proposition 1.5. Let $I$ be an ideal of $Q=k\left[X_{1}, \ldots, X_{n}\right]$ and $A=Q / I$ the quotient algebra. Denote by $\mathfrak{m}$ the maximal ideal $\left(X_{1}, \ldots, X_{n}\right)$ of 
$Q$. Then $\sqrt{I}=\mathfrak{m}$ and the $k$-algebra $A$ is Gorenstein if and only if there exists a polynomial $F \in R=k\left[x_{1}, \ldots, x_{n}\right]$ such that $I=\operatorname{Ann}_{Q} F$.

Example 1.6. The coinvariant algebra $R_{W}$ of the finite Coxeter group $W$ is an example of the finite-dimensional Gorenstein algebra with the strong Lefschetz property. The coinvariant algebra $R_{W}$ is defined to be a quotient of the ring of polynomial functions on the reflection representation $V$ of $W$ by the ideal generated by the fundamental $W$-invariants. When $W$ is crystallographic (i.e., Weyl group), the Lefschetz property of $R_{W}$ is a consequence of the Hard Lefschetz theorem for the corresponding flag variety $G / B$. Stanley [18] has shown the Sperner property of the strong Bruhat ordering on $W$ from the Lefschetz property of $R_{W}$ (except for type $H_{4}$ ). The Lefschetz property of $R_{W}$ of type $H_{4}$ has been confirmed in [15]. Since $R_{W}$ is Gorenstein, it has a presentation as in Proposition 1.5. In fact, $R_{W}$ is isomorphic to the algebra $\operatorname{Sym} V^{*} / \operatorname{Ann} F$, where $F$ is the product of the positive roots.

Definition 1.7. Let $G$ be a polynomial in $k\left[x_{1}, \ldots, x_{n}\right]$. When a family $\mathbf{B}_{d}=\left\{\alpha_{i}^{(d)}\right\}_{i}$ of homogeneous polynomials of degree $d>0$ is given, we call the polynomial

$$
\operatorname{det}\left(\left(\alpha_{i}^{(d)}(X) \alpha_{j}^{(d)}(X) G(x)\right)_{i, j=1}^{\# \mathbf{B}_{d}}\right) \in k\left[x_{1}, \ldots, x_{n}\right]
$$

the $d$ th Hessian of $G$ with respect to $\mathbf{B}_{d}$, and denote it by $\operatorname{Hess}_{\mathbf{B}_{d}}^{(d)} G$. We denote the $d$ th Hessian simply by $\operatorname{Hess}^{(d)} G$ if the choice of $\mathbf{B}_{d}$ is clear.

When $d=1$ and $\alpha_{j}^{(1)}(X)=X_{j}, j=1, \ldots, n$, the first Hessian Hess $^{(1)} G$ coincides with the usual Hessian:

$$
\operatorname{Hess}^{(1)} G=\operatorname{Hess} G:=\operatorname{det}\left(\frac{\partial^{2} G}{\partial x_{i} \partial x_{j}}\right)_{i j} .
$$

Let a finite-dimensional graded Gorenstein algebra $A=\oplus_{d} A_{d}$ have the presentation $A=Q / \operatorname{Ann}_{Q} F$. The following gives a criterion for an element $L \in A_{1}$ to be a Lefschetz element. 
Proposition 1.8. ([21, Theorem 4]). Fix an arbitrary $k$-linear basis $\mathbf{B}_{d}$ of $A_{d}$ for $d=1, \ldots,[D / 2]$. An element $L=a_{1} X_{1}+\cdots+a_{n} X_{n} \in$ $A_{1}$ is a strong Lefschetz element of $A=Q / \operatorname{Ann}_{Q} F$ if and only if $F\left(a_{1}, \ldots, a_{n}\right) \neq 0$ and

$$
\left(\operatorname{Hess}_{\mathbf{B}_{d}}^{(d)} F\right)\left(a_{1}, \ldots, a_{n}\right) \neq 0
$$

for $d=1, \ldots,[D / 2]$.

Corollary 1.9. If one of the Hessians $\operatorname{Hess}_{\mathbf{B}_{d}}^{(d)} F, d=1, \ldots,[D / 2]$, is identically zero, then $A=Q / \operatorname{Ann}_{Q} F$ does not have the strong Lefschetz property.

\section{Matroids.}

Definition 2.1. A pair $(E, \mathcal{F})$ of a finite set $E$ and $\mathcal{F} \subset 2^{E}$ is called a matroid if it satisfies the following axioms (M1), (M2) and (M3).

$(M 1) \emptyset \in \mathcal{F}$.

(M2) If $X \in \mathcal{F}$ and $Y \subset X$, then $Y \in \mathcal{F}$.

(M3) If $X, Y \in \mathcal{F}$ and $\# X>\# Y$, then there exists an element $x \in X \backslash Y$ such that $Y \cup\{x\} \in \mathcal{F}$. Here, $\mathcal{F}$ is called the system of independent sets.

Definition 2.2. Let $M=(E, \mathcal{F})$ be a matroid.

(1) A maximal element $B \in \mathcal{F}$ is called a basis of $M$. We denote by $\mathcal{B}=\mathcal{B}(M) \subset \mathcal{F}$ the set of bases of $M$.

(2) For a subset $S \subset E$, define $r(S):=\max \{\# F \mid F \in \mathcal{F}, F \subset S\}$. The map $r: 2^{E} \rightarrow \mathbb{Z}$ is called the rank function of $M$.

(3) For a subset $S \subset E$, define the closure $\sigma(S)$ of $S$ by

$$
\sigma(S):=\{y \in E \mid r(S \cup\{y\})=r(S)\} .
$$

We define an equivalence relation $\sim$ on $2^{E}$ by

$$
S \sim T \Longleftrightarrow \sigma(S)=\sigma(T) .
$$

A subset $S$ of $E$ is called a flat of $M$ if $S=\sigma(S)$.

Example 2.3. The projective space $\mathbb{P}:=\mathbb{P}^{n-1}\left(\mathbb{F}_{q}\right)$ over a finite field $\mathbb{F}_{q}$ has the structure of a matroid by the usual linear independence. 
More precisely, if we define the system of independence set $\mathcal{F}$ by

$$
\mathcal{F}:=\left\{F \in 2^{\mathbb{P}} \mid F \text { is linearly independent over } \mathbb{F}_{q}\right\},
$$

then $(\mathbb{P}, \mathcal{F})$ is a matroid. We denote it by $M(q, n)$. In this case, the closure $\sigma(S)$ of a subset $S \in \mathbb{P}$ coincides with the linear subspace $\langle S\rangle$ of $\mathbb{P}$ spanned by $S$.

Lemma 2.4. Let $S, T \in \mathcal{F}$. Then we have

$$
\begin{aligned}
S \sim T \Longleftrightarrow\{U \in \mathcal{F} \mid U \cap S=\emptyset, U \cup S \in \mathcal{F}\} \\
\quad=\{U \in \mathcal{F} \mid U \cap T=\emptyset, U \cup T \in \mathcal{F}\} .
\end{aligned}
$$

Proof. Let $S, U$ be independent sets. If $U \cap S=\emptyset$ and $S \cup U \in \mathcal{F}$, then $r(S \cup\{y\})=r(S)+1$ for all $y \in U$, and we have $U \cap \sigma(S)=\emptyset$. If $U \cap S=\emptyset$ and $S \cup U \notin \mathcal{F}$, then there exists an element $y \in U$ such that $r(S \cup\{y\})=r(S)$. So we have $U \cap \sigma(S) \neq \emptyset$. Hence, $\sigma(S)$ determines the set $\{U \in \mathcal{F} \mid U \cap S=\emptyset, U \cup S \in \mathcal{F}\}$, and vice versa.

Definition 2.5. For a given matroid $M=(E, \mathcal{F})$, the matroid polytope $P_{M} \subset \mathbb{R}^{E}$ is defined by the following system of inequalities:

$$
x_{e} \geq 0 \quad(e \in E), \quad \sum_{e \in A} x_{e} \leq r(A) \quad\left(A \in 2^{E}\right) .
$$

For each independent set $F \in \mathcal{F}$, we define the incidence vector $\vec{v}_{F}=\left(v_{F, e}\right)_{e \in E} \in \mathbb{R}^{E}$ as follows:

$$
v_{F, e}:= \begin{cases}1 & \text { if } e \in F \\ 0 & \text { otherwise }\end{cases}
$$

Proposition 2.6. (Edmonds [4]). The matroid polytope $P_{M}$ coincides with the convex hull of the union of $\{\overrightarrow{0}\}$ and the set of the incidence vectors of $\mathcal{F}$ :

$$
P_{M}=\operatorname{conv}\left(\{\overrightarrow{0}\} \cup\left\{\vec{v}_{F} \mid F \in \mathcal{F}\right\}\right) .
$$

Let $\Delta_{M}$ be the face of $P_{M}$ defined by the equation $\sum_{e \in E} x_{e}=r(E)$, which is also obtained as the convex hull of the incidence vectors corresponding to the bases of $M$. 
Example 2.7. Let $M$ be a matroid defined by the following vectors.

\begin{tabular}{c|c|c|c|c}
$v_{1}$ & $v_{2}$ & $v_{3}$ & $v_{4}$ & $v_{5}$ \\
1 & 0 & 0 & 1 & 0 \\
0 & 1 & 0 & 1 & 1 \\
0 & 0 & 1 & 0 & 1.
\end{tabular}

Then the basis of $M$ is $\mathcal{B}=\{\{1,2,3\},\{1,2,5\},\{1,3,4\},\{1,3,5\},\{1,4,5\}$, $\{2,3,4\},\{2,4,5\},\{3,4,5\}\}$. The polytope $\Delta_{M}$ is the convex hull of the following points in $\mathbb{R}^{5}$ :

$$
\begin{aligned}
& (1,1,1,0,0),(1,1,0,0,1),(1,0,1,1,0),(1,0,1,0,1), \\
& (1,0,0,1,1),(0,1,1,1,0),(0,1,0,1,1),(0,0,1,1,1) .
\end{aligned}
$$

3. Gorenstein algebras associated to matroids. For a matroid $M=(E, \mathcal{F})$, we define a polynomial $\Phi_{M} \in k\left[x_{e} \mid e \in E\right]$ by

$$
\Phi_{M}:=\sum_{B \in \mathcal{B}} x_{B},
$$

where $x_{B}:=\prod_{b \in B} x_{b}$. Note that the Newton polytope of $\Phi_{M}$ coincides with $\Delta_{M}$ in $\mathbb{R}^{E}$. Let $Q=Q_{M}=k\left[\partial / \partial x_{e} \mid e \in E\right]$ denote the ring of differential polynomials. For a subset $S \subset E$, we put $x_{S}:=\prod_{e \in S} x_{e}$ and $\partial^{S}:=\prod_{e \in S}\left(\partial / \partial x_{e}\right)$. In the subsequent part of this paper, we discuss the structure of the Gorenstein ring $A_{M}:=Q / \operatorname{Ann}_{Q} \Phi_{M}$.

Proposition 3.1. The ideal Ann $\Phi_{M}$ contains

$$
\begin{aligned}
\Lambda_{M} & :=\left\{x_{e}^{2} \mid e \in E\right\} \cup\left\{x_{S} \mid S \notin \mathcal{F}\right\} \\
& \cup\left\{x_{A}-x_{A^{\prime}} \mid A, A^{\prime} \in \mathcal{F}, A \sim A^{\prime}\right\} .
\end{aligned}
$$

Proof. Since $\Phi_{M}$ is square-free and does not contain the monomials of form $x_{S}, S \notin \mathcal{F}$, the ideal Ann $\Phi_{M}$ contains $\left\{x_{e}^{2} \mid e \in E\right\}$ and $\left\{x_{S} \mid S \notin \mathcal{F}\right\}$. If $A, A^{\prime} \in \mathcal{F}$ are equivalent, then we have $\partial^{A} \Phi_{M}=\partial^{A^{\prime}} \Phi_{M}$ from Lemma 2.4.

We denote by $J_{M} \subset Q$ the ideal generated by the set $\Lambda_{M}$. Let $M=(E, \mathcal{F})$ be a matroid, and $\mathcal{F}_{i} \subset \mathcal{F}$ for $i=1, \ldots, r(E)$ the set of independent sets of cardinality $i$, i.e.,

$$
\mathcal{F}_{i}:=\{F \in \mathcal{F} \mid \# F=i\}
$$


Let $\Omega:=2^{E} / \sim, \overline{\mathcal{F}}_{l}:=\mathcal{F}_{l} / \sim$ and $m_{l}:=\# \overline{\mathcal{F}}_{l}$. We can identify $\Omega$ with the set of the flats of $M$. Under this identification, we define the subset $\Omega(l), l=1, \ldots, r(E)$, of $\Omega$ by

$$
\Omega(l):=\left\{S \in 2^{E} \mid S=\sigma(S), r(S)=l\right\} .
$$

For an equivalence class $\tau \in \Omega$, consider a polynomial $f_{\tau}$ given by

$$
f_{\tau}:=\sum_{F \in \mathcal{F} \cap \tau} x_{F} .
$$

Proposition 3.2. We have

$$
J_{M}=\bigcap_{\tau \in \Omega} \operatorname{Ann} f_{\tau}
$$

Proof. It is easy to see that $\Lambda_{M}$ is contained in $\cap_{\tau \in \Omega} \operatorname{Ann} f_{\tau}$. It is enough to show that a polynomial $p \in \cap_{\tau \in \Omega}$ Ann $f_{\tau}$ of form

$$
p=\sum_{\tau \in \Omega} \sum_{F \in \mathcal{F} \cap \tau} a_{F} x_{F}, \quad a_{F} \in k,
$$

is a linear combination of polynomials of $\Lambda_{M}$. Put $p_{\tau}:=\sum_{F \in \mathcal{F} \cap \tau} a_{F} x_{F}$ and consider the polynomial

$$
p^{\prime}:=\sum_{\substack{\tau \in \Omega \\ p_{\tau} \notin \Lambda_{M}}} p_{\tau} .
$$

Choose $\tau_{0} \in \Omega$ with $p_{\tau} \neq 0$ of minimum rank. Then

$$
p(\partial) f_{\tau_{0}}=p_{\tau_{0}}(\partial) f_{\tau_{0}}=\sum_{F \in \mathcal{F} \cap \tau_{0}} a_{F}=0 .
$$

Let $\mathcal{F} \cap \tau=\left\{F_{1}, \ldots, F_{s}\right\}$. Then we have

$$
\begin{aligned}
p_{\tau}= & a_{F_{1}}\left(x_{F_{1}}-x_{F_{2}}\right)+\left(a_{F_{1}}+a_{F_{2}}\right)\left(x_{F_{2}}-x_{F_{3}}\right)+\cdots \\
& +\left(a_{F_{1}}+\cdots+a_{F_{s-1}}\right)\left(x_{F_{s-1}}-x_{F_{s}}\right) .
\end{aligned}
$$

Proposition 3.3. The subset $\Lambda_{M}$ of $Q$ is a universal Gröbner basis of $J_{M}$.

The proof is based on Buchberger's criterion. 
Proof. Fix a monomial ordering $\leq$ on the polynomial ring $Q$. For non-zero monic polynomials $f, g \in Q$, the $S$-polynomial $S(f, g)$ is given as follows:

$$
\begin{aligned}
& S(f, g):=-\frac{\Gamma(f, g)}{\operatorname{in}_{\leq}(f)} f+\frac{\Gamma(f, g)}{\operatorname{in}_{\leq}(g)} g, \\
& \Gamma(f, g):=\text { L. C. } \mathrm{M}\left(\operatorname{in}_{\leq}(f), \operatorname{in}_{\leq}(g)\right) .
\end{aligned}
$$

Let $\Lambda_{1}:=\left\{x_{A}-x_{A^{\prime}} \mid A, A^{\prime} \in \mathcal{F}, A \sim A^{\prime}\right\}$ and $\Lambda_{2}:=\left\{x_{e}^{2} \mid e \in E\right\}$ and $\Lambda_{3}:=\left\{x_{S} \mid S \notin \mathcal{F}\right\}$. We will show that the $S$-polynomials $S(f, g)$ are reduced to zero by the division algorithm with respect to $\Lambda_{M} \backslash\{f, g\}$ for cases:

(i) $f, g \in \Lambda_{1}$, (ii) $f \in \Lambda_{1}, g \in \Lambda_{2}$, (iii) $f \in \Lambda_{1}, g \in \Lambda_{3}$, (iv) $f, g \in \Lambda_{2} \cup \Lambda_{3}$.

Case (i). Take polynomials $f:=x_{A}-x_{A^{\prime}}$ and $g:=x_{B}-x_{B^{\prime}} \in \Lambda_{1}$ with $x_{A}>x_{A^{\prime}}$ and $x_{B}>x_{B^{\prime}}$. If $A \cap B=\emptyset$, it is easy to see that $S(f, g)$ is reduced to zero. Assume that $A \cap B \neq \emptyset$. Let $C:=A \cap B$, $\widehat{A}=A \backslash C$ and $\widehat{B}=B \backslash C$. Then we have $S(f, g)=x_{A^{\prime}} x_{\hat{B}}-x_{B^{\prime}} x_{\hat{A}}$. Note that we have

$$
\begin{aligned}
& r\left(A^{\prime} \cup \widehat{B}\right)=r(A \cup \widehat{B})=r(\widehat{A} \cup C \cup \widehat{B}), \\
& r\left(B^{\prime} \cup \widehat{A}\right)=r(B \cup \widehat{A})=r(\widehat{A} \cup C \cup \widehat{B}),
\end{aligned}
$$

so $r\left(A^{\prime} \cup \widehat{B}\right)=r\left(B^{\prime} \cup \widehat{A}\right)$.

(a) If $A^{\prime} \cap \widehat{B} \neq \emptyset$, then $x_{A^{\prime}} x_{\hat{B}} \in \Lambda_{2}$. In this case, we have

(*) $r\left(\widehat{A} \cup B^{\prime}\right)=r\left(A^{\prime} \cup \widehat{B}\right)<r\left(A^{\prime}\right)+r(\widehat{B})=\# A^{\prime}+\# \widehat{B}=\# \widehat{A}+\# B^{\prime}$,

which means that $\widehat{A} \cap B^{\prime} \neq \emptyset$ or $\widehat{A} \cup B^{\prime} \notin \mathcal{F}$. Hence, we also have $x_{\hat{A}} x_{B^{\prime}} \in \Lambda_{2} \cup \Lambda_{3}$.

(b) Assume that $A^{\prime} \cap \widehat{B}=\emptyset$. If $A^{\prime} \cup \widehat{B} \notin \mathcal{F}$, then we have $x_{A^{\prime}} x_{\hat{B}} \in \Lambda_{3}$. Moreover, again from the inequality $(*)$, we see that $x_{\hat{A}} x_{B^{\prime}} \in \Lambda_{2} \cup \Lambda_{3}$. If $A^{\prime} \cup \widehat{B} \in \mathcal{F}$, we have

$$
r\left(\widehat{A} \cup B^{\prime}\right)=r\left(A^{\prime} \cup \widehat{B}\right)=r\left(A^{\prime}\right)+r(\widehat{B})=\# A^{\prime}+\# \widehat{B}=\# \widehat{A}+\# B^{\prime},
$$

which means that $\widehat{A} \cup B^{\prime} \in \mathcal{F}$. Hence, we have $S(f, g)=x_{A^{\prime}} x_{\hat{B}}-$ $x_{B^{\prime}} x_{\hat{A}} \in \Lambda_{1}$.

Case (ii). Take polynomials $f:=x_{A}-x_{A^{\prime}} \in \Lambda_{1}$ and $g:=x_{e}^{2} \in \Lambda_{2}$ with $x_{A}>x_{A^{\prime}}$. If $e \notin A$, then $S(f, g)=x_{e}^{2} x_{A^{\prime}}$ is reduced to zero. If 
$e \in A$, then $S(f, g)=x_{e} x_{A^{\prime}}$. Since $r\left(A^{\prime} \cup\{e\}\right)=r(A \cup\{e\})=r(A)$, we have $x_{e} x_{A^{\prime}} \in \Lambda_{2} \cup \Lambda_{3}$.

Case (iii). Take polynomials $f:=x_{A}-x_{A^{\prime}} \in \Lambda_{1}$ and $g:=x_{B} \in \Lambda_{3}$ with $x_{A}>x_{A^{\prime}}$. If $A \cap B=\emptyset$, then $S(f, g)=x_{A^{\prime}} x_{B}$ is reduced to zero. If $A \cap B \neq \emptyset$, then $S(f, g)=x_{A^{\prime}} x_{B \backslash A}$. The inequality

$$
\begin{aligned}
r\left(A^{\prime} \cup(B \backslash A)\right) & =r(A \cup(B \backslash A))=r(A \cup B) \\
& <\#(A \cup B)=\#\left(A^{\prime} \cup(B \backslash A)\right)
\end{aligned}
$$

implies that $x_{A^{\prime}} x_{B \backslash A} \in \Lambda_{2} \cup \Lambda_{3}$.

Case (iv). This case is easy because $\Lambda_{2}$ and $\Lambda_{3}$ consist of monomials.

Corollary 3.4. The Hilbert function of $Q / J_{M}$ is given by

$$
\operatorname{Hilb}\left(Q / J_{M}, t\right)=\sum_{i=0}^{r(E)}\left(\# \overline{\mathcal{F}}_{i}\right) t^{i} .
$$

Example 3.5. Let $M$ be the matroid defined in Example 2.7. Then the ideal Ann $\Phi_{M}$ contains an additional generator other than $\Lambda_{M}$. In fact, we have

$$
\text { Ann } \Phi_{M}=J_{M}+\left(x_{13}+x_{45}-x_{15}-x_{34}\right) .
$$

The Hilbert series of $Q / \operatorname{Ann} \Phi_{M}$ is $(1,5,5,1)$ and that of $Q / J_{M}$ is $(1,5,6,1)$. In particular, $Q / J_{M}$ is not Gorenstein. By direct computation, we get

$$
\text { Hess } \Phi_{M}=8\left(x_{1}+x_{4}\right)\left(x_{3}+x_{5}\right) \Phi_{M} .
$$

This implies that $A_{M}=Q / \operatorname{Ann} \Phi_{M}$ has the strong Lefschetz property.

4. Vector space lattice. In this section, we treat the matroid $M=M(q, n)$ defined in Example 2.3. We define polynomials $\Phi_{M}^{(i)}:=$ $\sum_{G \in \mathcal{F}_{i}} x_{G}$ for $i=1, \ldots, n$. Note that $\Phi_{M}^{(n)}=\Phi_{M}$.

Lemma 4.1. For $M=M(q, n)$ and $l \leq[n / 2]$, the polynomials $\partial^{F} \Phi_{M}^{(2 l)}$, $F \in \overline{\mathcal{F}}_{l}$, are linearly independent over $k$. 
Proof. In the following, $\langle S\rangle$ stands for a linear subspace in $\mathbb{F}_{q}^{n}$ spanned by a subset $S \subset \mathbb{P}^{n-1}\left(\mathbb{F}_{q}\right)$. For $B \in \mathcal{F}_{l}$ and $0 \leq i \leq l$, define

$$
\mathcal{F}_{l}(B, i):=\left\{A \in \mathcal{F}_{l} \mid \operatorname{dim}(\langle A\rangle \cap\langle B\rangle)=i\right\}
$$

Then, we have

$$
\mathcal{F}_{l}(B, l)=\left\{A \in \mathcal{F}_{l} \mid A \sim B\right\}
$$

and

$$
\mathcal{F}_{l}=\bigcup_{i=0}^{l} \mathcal{F}_{l}(B, i)
$$

For $A, B \in \mathcal{F}_{l}$, we also define

$$
\begin{aligned}
\mathcal{F}_{l}^{A}(B, i) & :=\left\{A^{\prime} \in \mathcal{F}_{l}(B, i) \mid\langle A\rangle \cap\left\langle A^{\prime}\right\rangle=\{\overrightarrow{0}\}\right\} \\
& =\left\{A^{\prime} \in \mathcal{F}_{l}(B, i) \mid A \cup A^{\prime} \in \mathcal{F}_{2 l}\right\} .
\end{aligned}
$$

For $B \in \mathcal{F}_{l}$, consider a polynomial $\Phi(B, i):=\sum_{A \in \mathcal{F}_{l}(B, i)} x_{A}$ and a differential polynomial $P(B, i):=\sum_{A \in \mathcal{F}_{l}(B, i)} \partial^{A}$. We have

$$
\begin{aligned}
P(B, i) \Phi_{M}^{(2 l)} & =\sum_{A \in \mathcal{F}_{l}(B, i)} \partial^{A} \Phi_{M}^{(2 l)}=\sum_{A \in \mathcal{F}_{l}(B, i)} \sum_{\substack{A^{\prime} \in \mathcal{F}_{l} \\
A \cup A^{\prime} \in \mathcal{F}_{2 l}}} x_{A^{\prime}} \\
& =\sum_{A^{\prime} \in \mathcal{F}_{l}} \sum_{\substack{A \in \mathcal{F}_{l}(B, i) \\
A \cup A^{\prime} \in \mathcal{F}_{2 l}}} x_{A^{\prime}} \\
& =\sum_{j=0}^{l} \sum_{A^{\prime} \in \mathcal{F}_{l}(B, j)} \#\left\{A \in \mathcal{F}_{l}(B, i) \mid A \cup A^{\prime} \in \mathcal{F}_{2 l}\right\} x_{A^{\prime}} \\
& =\sum_{j=0}^{l} \sum_{A^{\prime} \in \mathcal{F}_{l}(B, j)} \# \mathcal{F}_{l}^{A^{\prime}}(B, i) x_{A^{\prime}} .
\end{aligned}
$$

Here, $\# \mathcal{F}_{l}^{A^{\prime}}(B, i)$ is independent of the choice of $A^{\prime} \in \mathcal{F}_{l}(B, j)$ for $M=M(q, n)$. Put $a_{i j}^{B}:=\# \mathcal{F}_{l}^{A^{\prime}}(B, i)$ for $B \in \mathcal{F}_{l}$ and $A^{\prime} \in \mathcal{F}_{l}(B, j)$. Now we have

$$
P(B, i) \Phi_{M}^{(2 l)}=\sum_{j=1}^{l} a_{i j}^{B} \sum_{A^{\prime} \in \mathcal{F}_{l}(B, j)} x_{A^{\prime}}=\sum_{j=1}^{l} a_{i j}^{B} \Phi(B, j) .
$$


If $i+j>l$, then $\operatorname{dim}(\langle A\rangle \cap\langle B\rangle)+\operatorname{dim}\left(\left\langle A^{\prime}\right\rangle \cap\langle B\rangle\right)=i+j>l$. Hence, we have $\operatorname{dim}\left(\langle A\rangle \cap\left\langle A^{\prime}\right\rangle \cap\langle B\rangle\right)>0$ and $\langle A\rangle \cap\left\langle A^{\prime}\right\rangle \neq\{\overrightarrow{0}\}$. This means that $a_{i j}^{B}=\# \mathcal{F}_{l}^{A^{\prime}}(B, i)=0$.

Assume that $i+j=l$. For $A \in \mathcal{F}_{l}(B, j)$, take an element $A_{1} \in \mathcal{F}_{j}$ such that $\left\langle A_{1}\right\rangle=\langle A\rangle \cap\langle B\rangle$. We also take an element $A_{2} \in \mathcal{F}_{l-j}=\mathcal{F}_{i}$ such that $\left\langle A_{1} \cup A_{2}\right\rangle=\langle B\rangle$, and $A_{3} \in \mathcal{F}_{n-l}$ such that $\left\langle B \cup A_{3}\right\rangle=\mathbb{F}_{q}^{n}$. Put $A^{*}:=A_{2} \cup A_{3}$. Since $\operatorname{dim}\left\langle A^{*}\right\rangle=n-j \geq n-l \geq l$, there exists an element $A^{\prime} \in \mathcal{F}_{l}$ such that $\left\langle A^{*}\right\rangle \cap\langle B\rangle \subset\left\langle A^{\prime}\right\rangle \subset\left\langle A^{*}\right\rangle$. Since $\left\langle A^{\prime}\right\rangle \cap\langle B\rangle=\left\langle A^{*}\right\rangle \cap\langle B\rangle=\left\langle A_{2}\right\rangle$, we can see that $A^{\prime} \in \mathcal{F}_{l}^{A}(B, i)$. Hence, we have $a_{i j}^{B}>0$ in this case.

We have seen that the matrix $\left(a_{i, l-j}^{B}\right)_{i, j=0}^{l}$ is upper-triangular, so

$$
\operatorname{det}\left(a_{i, l-j}^{B}\right)_{i j}=\prod_{i=0}^{l} a_{i, l-i}^{B}>0 .
$$

Since the matrix $\left(a_{i, l-j}\right)_{i j}$ is invertible, $\Phi_{M}(B, l)$ may be written as a linear combination of $P(B, 0) \Phi_{M}^{(2 l)}, P(B, 1) \Phi_{M}^{(2 l)}, \ldots, P(B, l) \Phi_{M}^{(2 l)}$, and hence it is a linear combination of the polynomials $\partial^{F} \Phi_{M}^{(2 l)}, F \in \overline{\mathcal{F}}_{l}$. On the other hand, it is easy to see the linear independence of the polynomials $\Phi_{M}(B, l)$ for $B \in \overline{\mathcal{F}}_{l}$. Therefore, the polynomials $\partial^{F} \Phi_{M}^{(2 l)}$ for $F \in \overline{\mathcal{F}}_{l}$, are linearly independent.

Proposition 4.2. Let $M=M(q, n)$. Take a representative $F_{1}, \ldots, F_{m_{l}}$ $\in \mathcal{F}_{l}$ of $\overline{\mathcal{F}}_{l}$. Then the determinant of the matrix

$$
\left(\partial^{F_{i}} \partial^{F_{j}} \Phi_{M}\right)_{i, j=1}^{m_{l}}
$$

is not identically zero.

Proof. For $F \in \mathcal{F}_{j}$, define $c(F, i):=\#\left\{F^{\prime} \in \mathcal{F}_{i} \mid F \cup F^{\prime} \in \mathcal{F}_{i+j}\right\}$. Then the equality $c\left(F_{1}, i\right)=c\left(F_{2}, i\right)$ holds for any $F_{1}, F_{2} \in \mathcal{F}_{j}$ and for $j=1, \ldots, r(E)-1$. It is easy to see that

$$
\left.\operatorname{det}\left(\partial^{F_{i}} \partial^{F_{j}} \Phi_{M}\right)_{i, j=1}^{m_{l}}\right|_{x=1}=\gamma \cdot \operatorname{det}\left(\delta_{\sigma\left(F_{i}\right), \sigma\left(F_{j}\right)}\right)_{i, j},
$$

where $\gamma=c(F, l)^{m_{l}} \neq 0$ for any $F \in \mathcal{F}_{l}$, and $\delta_{\tau_{1}, \tau_{2}}, \tau_{1}, \tau_{2} \in \Omega(l)$, is 
defined by

$$
\delta_{\tau_{1}, \tau_{2}}:= \begin{cases}1 & \text { if } \tau_{1} \cap \tau_{2}=\emptyset \\ 0 & \text { otherwise }\end{cases}
$$

At the same time, we have

$$
\operatorname{det}\left(\partial^{F_{i}} \partial^{F_{j}} \Phi_{M}^{(2 l)}\right)_{i, j}=\operatorname{det}\left(\delta_{\sigma\left(F_{i}\right), \sigma\left(F_{j}\right)}\right)_{i, j} .
$$

Note that the algebra $B^{(2 l)}:=Q / \operatorname{Ann} \Phi_{M}^{(2 l)}$ is also Gorenstein, and the natural pairings

$$
\langle,\rangle: B_{i}^{(2 l)} \times B_{2 l-i}^{(2 l)} \longrightarrow B_{2 l}^{(2 l)} \cong k
$$

are non-degenerate for $i=0, \ldots, l$. From Lemma 4.1 , we see that $\left\{x_{F_{i}} \mid\right.$ $\left.i=1, \ldots, m_{l}\right\}$ gives a basis of $B_{l}^{(2 l)}$. Since the matrix $\left(\partial^{F_{i}} \partial^{F_{j}} \Phi_{M}^{(2 l)}\right)_{i, j}$ represents the pairing $\langle$,$\rangle at the intermediate part B_{l}^{(2 l)} \times B_{l}^{(2 l)} \rightarrow k$, we see that its determinant is non-zero. Therefore, $\left.\operatorname{det}\left(\partial^{F_{i}} \partial^{F_{j}} \Phi_{M}\right)\right|_{x=1}$ is non-zero, and hence it cannot be identically zero.

\section{Theorem 4.3.}

(1) The algebra $A_{M(q, n)}$ has the strong Lefschetz property.

(2) The ideal Ann $\Phi_{M(q, n)}$ is generated by $\Lambda_{M(q, n)}$, i.e., Ann $\Phi_{M(q, n)}=$ $J_{M(q, n)}$. In particular, it is a binomial ideal.

(3) We have

$$
\operatorname{Hilb}\left(Q / \operatorname{Ann} \Phi_{M(q, n)}, t\right)=\sum_{i=0}^{n} t^{i}\left(\begin{array}{l}
n \\
i
\end{array}\right)_{q},
$$

where $\left(\begin{array}{c}n \\ i\end{array}\right)_{q}, 0 \leq i \leq n$, are $q$-binomial coefficients.

(4) The vector space lattice $V(q, n)$ consisting of the linear subspaces of $\mathbb{F}_{q}^{n}$ has the Sperner property.

Proof. Proposition 4.2 implies that the monomials $X_{F_{i}}, i=1, \ldots, m_{l}$, are linearly independent in the algebra $A_{M}$. Hence, we get $Q / J_{M}=$ $A_{M}$ by comparing their dimension over $k$. This shows (2) and (3). Since the monomials $X_{F_{i}}, i=1, \ldots, m_{l}$, form a linear basis of $\left(A_{M}\right)_{l}$, Proposition 4.2 also implies that the polynomial

$$
\operatorname{Hess}_{\left\{X_{F_{i}}\right\}_{i=1}^{m_{l}}}^{(l)} \Phi_{M(q, n)}=\operatorname{det}\left(\partial^{F_{i}} \partial^{F_{j}} \Phi_{M}\right)_{i, j=1}^{m_{l}}
$$


is a nonzero polynomial for $l=1, \ldots,[n / 2]$. Then we have the strong Lefschetz property for $A_{M}$ by Proposition 1.8. The deduction of the Sperner property for $M$ from the Lefschetz property is a standard argument (see, e.g., $[\mathbf{1 0}, 1.4 .1]$ for details.)

Remark 4.4. For $i \leq n$, let $M^{(i)}(q, n)$ be a matroid structure on $\mathbb{P}^{n-1}\left(\mathbb{F}_{q}\right)$ obtained by regarding $\mathcal{F}_{i}$ as a system of bases. We see that $\Phi_{M^{(i)}(q, n)}=\Phi_{M(q, n)}^{(i)}$. It can be shown by a similar manner as the proof of Proposition 4.2 that $Q / \operatorname{Ann} \Phi_{M^{(i)}(q, n)}$ has the Lefschetz property.

Example 4.5. Let $[n]:=\{1,2, \ldots, n\}$ be an $n$-element set. The set $2^{[n]}$ of the subsets of $[n]$ has a natural lattice structure induced by the operations $\cup$ and $\cap$. This lattice is called the Boolean lattice. Sperner's theory originates his work [17] on the maximal cardinality of the antichains of the Boolean lattice. On the other hand, $M([n]):=$ $\left([n], 2^{[n]}\right)$ satisfies the axioms for a matroid. The matroid $M([n])$ has the unique basis $[n]$, so the corresponding Gorenstein algebra is given by

$$
A_{M([n])}=k\left[X_{1}, \ldots, X_{n}\right] / \operatorname{Ann}\left(x_{1} \cdots x_{n}\right)=k\left[X_{1}, \ldots, X_{n}\right] /\left(X_{1}^{2}, \ldots, X_{n}^{2}\right) .
$$

In [9], it has been proved that $M([n])$ is another example of matroids for which Proposition 4.2 holds. As a consequence, we obtain Ann $\Phi_{M([n])}=J_{M([n])}$ and the Lefschetz property for $A_{M([n])}$, which gives another proof of the Sperner property for the Boolean lattice.

5. Modular geometric lattice. In this section, we discuss a characterization of the matroids for which the algebra $Q / J_{M}$ is Gorenstein.

Definition 5.1. Let $L$ be a finite graded lattice with the rank function $r$.

(1) The lattice $L$ is called (upper) semimodular if $r(x)+r(y) \geq$ $r(x \wedge y)+r(x \vee y)$ for all $x, y \in L$. If the equality holds for all $x, y \in L$, then $L$ is called modular.

(2) Assume that $L$ has the unique minimal element $\hat{0}$. An element of $L$ is called an atom if it covers $\hat{0}$. The term coatom is dually defined as an element covered by the unique maximal element $\hat{1}$. 
The lattice $L$ is atomic if every element of $L$ is written as a join of atoms.

(3) The lattice $L$ is said to be geometric if $L$ is atomic and semimodular.

The set of the flats of a matroid forms a lattice, which we denote by $L(M)$. It is known that a finite lattice $L$ is geometric if and only if $L \cong L(M)$ for a matroid $M$ (see [19, Theorem 3.8]).

Proposition 5.2. (Greene [8]). Let L be a finite geometric lattice. The sets of atoms and of coatoms have the same cardinality if and only if $L$ is modular.

Greene's characterization of the modular geometric lattice implies the following.

Proposition 5.3. If $Q / J_{M}$ is Gorenstein, then $L(M)$ is a modular geometric lattice.

Proof. Let $n$ be the dimension of $M$. Then the socle degree of $Q / J_{M}$ is $n$. Suppose that $Q / J_{M}$ is Gorenstein. From Proposition 1.3, the part $\left(Q / J_{M}\right)_{1}$ of degree 1 is isomorphic to $\left(Q / J_{M}\right)_{n-1}$ of degree $n-1$ as vector spaces. Since

$$
\begin{aligned}
\#\{\text { atoms of } L(M)\} & =\operatorname{dim}\left(Q / J_{M}\right)_{1}=\operatorname{dim}\left(Q / J_{M}\right)_{n-1} \\
& =\#\{\text { coatoms of } L(M)\}
\end{aligned}
$$

we can conclude that the lattice $L(M)$ is a modular geometric lattice by Proposition 5.2.

The fundamental theorem of projective geometry shows that a modular geometric lattice decomposes into a direct product of boolean lattices, vector space lattices, lattices of rank 2 and incidence lattices of (non-Desarguesian) finite projective planes (see, e.g., [19]).

Proposition 5.4. Let $M(\Pi)$ be the matroid associated to a finite projective plane $\Pi$. Then we have $J_{M(\Pi)}=\operatorname{Ann} \Phi_{M(\Pi)}$.

Proof. Let $\Pi$ be a projective plane of order $\nu$. Since $J_{M(\Pi)} \subset$ Ann $\Phi_{M(\Pi)}$, we have a surjective homomorphism $\varphi: Q / J_{M(\Pi)} \rightarrow$ 
$A_{M(\Pi)}$. From Corollary 3.4, we have $\operatorname{dim}\left(Q / J_{M(\Pi)}\right)_{1}=\operatorname{dim}\left(Q / J_{M(\Pi)}\right)_{2}$ $=\nu^{2}+\nu+1$. Hence, in order to show that $\varphi$ is an isomorphism, it is enough to show $\operatorname{dim}\left(Q / J_{M(\Pi)}\right)_{1}=\operatorname{dim}\left(A_{M(\Pi)}\right)_{1}$. For two distinct points $p, q \in \Pi$, denote by $L_{p q}$ the line passing through $p$ and $q$. We have

$$
\partial^{p} \partial^{q} \Phi_{M(\Pi)}=\sum_{r \notin L_{p q}} x_{r},
$$

for $p \neq q$. Consider the specialization $S$ of the matrix $\left(\partial^{p} \partial^{q} \Phi_{M(\Pi)}\right)_{p, q \in \Pi}$ at $x_{a}=1$ for all $a \in \Pi$. Then we have

$$
S_{p q}= \begin{cases}0 & \text { if } p=q, \\ \nu^{2} & \text { if } p \neq q,\end{cases}
$$

and $\operatorname{det} S \neq 0$. So the polynomials $\partial^{p} \Phi_{M(\Pi)}, p \in \Pi$, are linearly independent. This shows $\operatorname{dim}\left(Q / J_{M(\Pi)}\right)_{1}=\operatorname{dim}\left(A_{M(\Pi)}\right)_{1}$.

Corollary 5.5. The algebra $A_{M(\Pi)}$ has the strong Lefschetz property.

The following lemma is easy.

Lemma 5.6. If $M$ is the direct sum of two matroids $M_{1}$ and $M_{2}$, then $Q_{M} / J_{M} \cong Q_{M_{1}} / J_{M_{1}} \otimes Q_{M_{2}} / J_{M_{2}}$.

Theorem 5.7. The algebra $Q / J_{M}$ is Gorenstein if and only if $L(M)$ is a modular geometric lattice.

Proof. In Proposition 5.3, we have proved that $L(M)$ is a modular geometric lattice if $Q / J_{M}$ is Gorenstein.

Conversely, assume that $L(M)$ is a modular geometric lattice. Then $L(M)$ decomposes into a direct product of boolean lattices $2^{[n]}$, vector space lattices $V(q, n)=L(M(q, n))$ and incidence lattices of finite projective planes $\Pi$. For the boolean lattice $2^{[n]}$, we have seen in Example 4.5 that $Q / J_{M([n])}$ is Gorenstein. For the matroid $M(q, n)$, it has been shown in Theorem $4.3(2)$ that $J_{M(q, n)}=\operatorname{Ann} \Phi_{M(q, n)}$, so $Q / J_{M(q, n)}$ is Gorenstein. In Proposition 5.4, we see that $Q / J_{M(\Pi)}$ is Gorenstein for a finite projective plane $\Pi$. Hence, from Corollary 1.4 and Lemma 5.6, the algebra $Q / J_{M}$ is Gorenstein. 


\section{Corollary 5.8.}

(1) The ideal $J_{M}$ coincides with Ann $\Phi_{M}$ if and only if $L(M)$ is a modular geometric lattice.

(2) If $L(M)$ is a modular geometric lattice, then $A_{M}$ has the strong Lefschetz property.

(3) Every modular geometric lattice has the Sperner property.

6. Gröbner fan of $J_{M}$. In this section, we discuss the Gröbner fan of the ideals $J_{M}$ and $\operatorname{Ann} \Phi_{M(q, n)}$. The initial ideal $\operatorname{in}_{\vec{\omega}}(I)$ of an ideal $I \subset Q$ with respect to the weight vector $\vec{\omega} \in \mathbb{R}^{E}$ is given by

$$
\operatorname{in}_{\vec{\omega}}(I):=\left(\operatorname{in}_{\vec{\omega}}(f) \mid f \in I, f \neq 0\right) .
$$

For a weight vector $\vec{\omega}$, the set $C(\vec{\omega}):=\operatorname{closure}\left\{\vec{\lambda} \in \mathbb{R}^{E} \mid \operatorname{in}_{\vec{\lambda}}(I)=\right.$ $\left.\operatorname{in}_{\vec{\omega}}(I)\right\}$ is a polyhedral cone in $\mathbb{R}^{E}$. The set of cones $\{C(\vec{\omega}) \mid \vec{\omega} \in$ $\left.\mathbb{R}^{E} \backslash\{\overrightarrow{0}\}\right\}$ forms a fan $G(I)$. The fan $G(I)$ is called the Gröbner fan of $I$. Denote by $G^{d}(I)$ the set of $d$-dimensional cones in $G(I)$. The Gröbner fan $G(I)$ of a homogeneous ideal $I$ has the translation invariance in the direction of $\vec{n}:=(1, \ldots, 1) \in \mathbb{R}^{E}$. Let $H$ be the hyperplane in $\mathbb{R}^{E}$ defined by the equation $\sum_{e \in E} x_{e}=0$. Denote by $\bar{G}(I)$ the restriction of $G(I)$ to $H$.

For two distinct independent sets $F, F^{\prime} \in \mathcal{F}$ with $F \sim F^{\prime}$, define a cone $W_{F, F^{\prime}}$ by the condition

$$
\begin{gathered}
\sum_{e \in F} x_{e}=\sum_{e \in F^{\prime}} x_{e}, \quad \sum_{e \in F} x_{e} \leq \sum_{e \in F^{\prime \prime}} x_{e} \\
\text { (for all } \left.F^{\prime \prime} \in \mathcal{F}, F^{\prime \prime} \sim F\right) .
\end{gathered}
$$

Let $C_{1}, \ldots, C_{p}$ be the closures of the connected components of

$$
\mathbb{R}^{E} \backslash \bigcup_{\substack{F, F^{\prime} \in \mathcal{F} \\ F \sim F^{\prime} \\ F \neq F^{\prime}}} W_{F, F^{\prime}} .
$$

Proposition 6.1. The maximal cones of $G\left(J_{M}\right)$ are given by $C_{1}, \ldots, C_{p}$, i.e., $G^{\# E}\left(J_{M}\right)=\left\{C_{1}, \ldots, C_{p}\right\}$.

Proof. Since $\Lambda_{M}$ is a universal Gröbner basis of $J_{M}, \operatorname{in}_{\vec{\omega}}\left(J_{M}\right)$ is not a monomial ideal if and only if $\operatorname{in}_{\vec{\omega}}\left(J_{M}\right)$ contains $x_{F}-x_{F^{\prime}}$ for two 
distinct independent sets $F, F^{\prime}$ with $F \sim F^{\prime}$ and does not contain $x_{F}$ or $x_{F^{\prime}}$. This is the case when $\vec{\omega} \in W_{F, F^{\prime}}$.

The tropical hypersurface $V_{\text {trop }}\left(\Phi_{M}\right) \subset \mathbb{R}^{E}$ is defined as the locus in $\mathbb{R}^{E}$ where the piecewise linear function

$$
\operatorname{trop}\left(\Phi_{M}\right)=\max \left(\sum_{e \in B} x_{e} \mid B \in \mathcal{B}\right)
$$

is not smooth. The tropical hypersurface $V_{\text {trop }}\left(\Phi_{M}\right)$ can be considered as a subcomplex of $G\left(\Phi_{M}\right)$ (see [2]). Since $\Phi_{M}$ is homogeneous, the corresponding tropical hypersurface $V_{\text {trop }}\left(\Phi_{M}\right)$ has the translation invariance in the direction of the vector $\vec{n}$. Denote by $\bar{V}_{\text {trop }}\left(\Phi_{M}\right)$ the restriction of $V_{\text {trop }}\left(\Phi_{M}\right)$ to $H$. In our case, $\bar{V}_{\text {trop }}\left(\Phi_{M}\right)$ is also regarded as a fan. The following proposition shows that the tropical variety $\bar{V}_{\text {trop }}\left(\Phi_{M}\right)$ is directly obtained from the matroid polytope of $M$.

Proposition 6.2. The piecewise linear function $\left.\operatorname{trop}\left(\Phi_{M}\right)\right|_{H}$ is a support function for the polytope $\Delta_{M}^{0}:=\Delta_{M}-r(E)(\# E)^{-1} \cdot \vec{n} \subset H$.

Proof. The polytope $\Delta_{M}^{0}$ is spanned by the vectors $\vec{u}_{B}:=\vec{v}_{B}-$ $r(E)(\# E)^{-1} \cdot \vec{n}, B \in \mathcal{B}$, by Proposition 2.6. We also have the inequality

$$
\left\langle\vec{u}_{B}, \vec{y}\right\rangle=\sum_{b \in B} y_{b} \leq \operatorname{trop}\left(\Phi_{M}\right)(\vec{y}), \quad \text { for all } \vec{y}=\left(y_{e}\right)_{e \in E} \in H,
$$

and, for $\vec{y}=\vec{u}_{B}$,

$$
\left\langle\vec{u}_{B}, \vec{u}_{B}\right\rangle=r(E)-\frac{r(E)^{2}}{\# E}=\operatorname{trop}\left(\Phi_{M}\right)\left(\vec{u}_{B}\right) .
$$

Hence, the polytope $\Delta_{M}^{0}$ is described as

$$
\Delta_{M}^{0}=\left\{\vec{x} \in H \mid\langle\vec{x}, \vec{y}\rangle \leq \operatorname{trop}\left(\Phi_{M}\right)(\vec{y}), \quad \text { for all } \vec{y} \in H\right\} .
$$

For a fan $\Sigma$, define $-\Sigma:=\{-\sigma \mid \sigma \in \Sigma\}$.

\section{Proposition 6.3.}

(1) For an equivalence class $\tau \in \Omega(l)$ with $l \geq 2$, we have

$$
G^{\# E-1}\left(f_{\tau}\right)=\left\{-W_{F, F^{\prime}} \mid F, F^{\prime} \in \mathcal{F} \cap \tau, F \neq F^{\prime}\right\} .
$$


(2)

$$
V_{\text {trop }}\left(f_{\tau}\right)=\bigcup_{\sigma \in G^{\# E-1}\left(f_{\tau}\right)} \sigma=\bigcup_{\substack{F, F^{\prime} \in \mathcal{F} \cap \tau \\ F \neq F^{\prime}}}-W_{F, F^{\prime}}
$$

(3) The fan $-G\left(J_{M}\right)$ is the common refinement of the fans defined by the tropical hypersurfaces $V_{\text {trop }}\left(f_{\tau}\right), \tau \in \Omega$.

Proof. Since the Newton polytope of $f_{\tau}$ does not contain interior lattice points, every monomial $x_{F}, F \in \mathcal{F} \cap \tau$, appearing in $f_{\tau}$, can be the initial monomial for a choice of monomial ordering. Hence, $\operatorname{in}_{\vec{\omega}}\left(f_{\tau}\right)$ is not a monomial ideal if $\vec{\omega}$ belongs to $-W_{F, F^{\prime}}$ for a pair $F, F^{\prime} \in \mathcal{F} \cap \tau$, $F \neq F^{\prime}$. This shows (1).

The second claim (2) follows from the definition of the tropical hypersurface $V_{\text {trop }}\left(f_{\tau}\right)$.

Claim (3) is a consequences of (2) and Proposition 6.1.

Corollary 6.4. The tropical hypersurface $V_{\text {trop }}\left(\Phi_{M}\right)$ is a subcomplex of the fan $-G\left(J_{M}\right)$.

For $M=M(q, n)$, we have $G\left(\operatorname{Ann} \Phi_{M(q, n)}\right)=G\left(J_{M(q, n)}\right)$ from Corollary 4.3 (2). By Proposition 6.3, the Gröbner fan $G\left(\right.$ Ann $\left.\Phi_{M(q, n)}\right)$ can be computed from the tropical hypersurfaces $V_{\text {trop }}\left(f_{\tau}\right)$.

Example 6.5. The matroid $M(2,2)$ is defined by the following three vectors:

\begin{tabular}{c|c|c}
$v_{1}$ & $v_{2}$ & $v_{3}$ \\
1 & 0 & 1 \\
0 & 1 & 1
\end{tabular}

so we have

$$
\begin{aligned}
& \Phi_{M(2,2)}=x_{1} x_{2}+x_{1} x_{3}+x_{2} x_{3}, \\
& \text { Ann } \Phi_{M(2,2)}=\left(x_{1}^{2}, x_{2}^{2}, x_{3}^{2}, x_{1} x_{2}-x_{1} x_{3}, x_{1} x_{2}-x_{2} x_{3}, x_{1} x_{3}-x_{2} x_{3}\right) .
\end{aligned}
$$

In this case, Gröbner fans $G\left(\operatorname{Ann} \Phi_{M(2,2)}\right), G\left(J_{M(2,2)}\right)$ and $-G\left(\Phi_{M(2,2)}\right)$ are the same. Their restrictions $\bar{G}\left(\operatorname{Ann} \Phi_{M(2,2)}\right), \bar{G}\left(J_{M(2,2)}\right)$ and 
$-\bar{G}\left(\Phi_{M(2,2)}\right)$ to the plane $H$ are determined by three rays:

$$
\begin{aligned}
& R_{1}:=\mathbb{R}_{\geq 0}(-2,1,1), \\
& R_{2}:=\mathbb{R}_{\geq 0}(1,-2,1), \\
& R_{3}:=\mathbb{R}_{\geq 0}(1,1,-2) .
\end{aligned}
$$

Moreover, $\bar{V}_{\text {trop }}\left(\Phi_{M(2,2)}\right)=\left(-R_{1}\right) \cup\left(-R_{2}\right) \cup\left(-R_{3}\right)$.

The following examples are obtained by using Sage [20].

Example 6.6. The Gröbner fan $\bar{G}\left(\operatorname{Ann} \Phi_{M(2,3)}\right)=\bar{G}\left(J_{M(2,3)}\right)$ contains 420 cones of maximal dimension 6 and 49 rays. The fan $\bar{G}\left(\Phi_{M(2,3)}\right)$ contains 28 maximal cones and 21 rays.

Example 6.7. Let $M$ be the matroid from Example 2.7. The fan $\bar{G}\left(J_{M}\right)$ contains 12 cones of maximal dimension 4 and 7 rays:

$$
\begin{array}{ll}
\mathbb{R}_{\geq 0}(-4,1,1,1,1), & \mathbb{R}_{\geq 0}(-2,-2,3,-2,3), \\
\mathbb{R}_{\geq 0}(-1,4,-1,-1,-1), & \mathbb{R}_{\geq 0}(1,1,-4,1,1), \\
\mathbb{R}_{\geq 0}(1,1,1,-4,1), & \mathbb{R}_{\geq 0}(1,1,1,1,-4), \\
\mathbb{R}_{\geq 0}(3,-2,-2,3,-2) . &
\end{array}
$$

The fan $\bar{G}\left(\Phi_{M}\right)$ contains eight maximal cones, and $\bar{G}^{1}\left(\Phi_{M}\right)=-\bar{G}^{1}\left(J_{M}\right)$. In this case, $\bar{G}\left(\operatorname{Ann} \Phi_{M}\right)$ is a refinement of $\bar{G}\left(J_{M}\right)$. The fan $\bar{G}\left(\operatorname{Ann} \Phi_{M}\right)$ contains 20 maximal cones and 9 rays:

$$
\begin{array}{ll}
\mathbb{R}_{\geq 0}(-4,1,1,1,1), & \mathbb{R}_{\geq 0}(-3,2,2,-3,2), \\
\mathbb{R}_{\geq 0}(-2,-2,3,-2,3), & \mathbb{R}_{\geq 0}(-1,4,-1,-1,-1), \\
\mathbb{R}_{\geq 0}(1,1,-4,1,1), & \mathbb{R}_{\geq 0}(1,1,1,-4,1), \\
\mathbb{R}_{\geq 0}(1,1,1,1,-4), & \mathbb{R}_{\geq 0}(2,2,-3,2,-3), \\
\mathbb{R}_{\geq 0}(3,-2,-2,3,-2) . &
\end{array}
$$

Acknowledgments. The authors thank Junzo Watanabe for suggesting the idea of the proof of the Sperner property for the vector space lattice via the Lefschetz property. They are grateful to Satoshi Murai for his helpful comment on modular geometric lattices. They also appreciate the anonymous referee's suggestions. 


\section{REFERENCES}

1. K.A. Baker, A generalization of Sperner's lemma, J. Comb. Th. 6 (1969), $224-225$.

2. T. Bogart, A.N. Jensen, D. Speyer, B. Sturmfels and R.R. Thomas, Computing tropical varieties, J. Symb. Comp. 42 (2007), 54-73.

3. W. Bruns and J. Herzog, Cohen-Macauley rings, Cambr. Stud. Adv. Math. 39, Cambridge University Press, Cambridge, 1993.

4. J. Edmonds, Submodular functions, matroids, and certain polyhedra, in Combinatorial structures and their applications, Gordon and Breach, New York 1970, 69-70.

5. A.V. Geramita, Inverse systems of fat points: Waring's problem, secant varieties of Veronese varieties and parameter spaces for Gorenstein ideals, in The curves seminar at Queen's, Vol. X, Queen's Papers Pure Appl. Math. 102 (1996), $2-114$.

6. A.V. Geramita, T. Harima, J.C. Migliore and Y.S. Shin, The Hilbert function of a level algebra, Mem. Amer. Math. Soc. 186 (2007), 139 pages.

7. S. Goto and K. Watanabe, On graded rings I, J. Math. Soc. Japan 30 (1978), $179-213$.

8. C. Greene, A rank inequality for finite geometric lattices, J. Comb. Th. 9 (1970), 357-364.

9. M. Hara and J. Watanabe, The determinants of certain matrices arising from the Boolean lattice, Discr. Math. 308 (2008), 5815-5822.

10. T. Harima, T. Maeno, H. Morita, Y. Numata, A. Wachi and J. Watanabe, The Lefschetz properties, LNM 2080, Springer, 2013.

11. W.M. Kantor, On incidence matrices of finite projective and affine spaces, Math. Z. 124 (1972), 315-318.

12. T. Maeno and Y. Numata, Sperner property, matroids and finitedimensional Gorenstein algebras, Contemp. Math. 580, American Mathematical Society, Providence, RI, 2012.

13. On the Sperner property and Gorenstein algebras associated to matroids, DMTCS Proc. 2012, 157-168.

14. T. Maeno and J. Watanabe, Lefschetz elements of Artinian Gorenstein algebras and Hessians of homogeneous polynomials, Illinois J. Math. 53 (2009), 591-603.

15. Y. Numata and A. Wachi, The strong Lefschetz property of the coinvariant ring of the Coxeter group of type $H_{4}$, J. Alg. 318 (2007), 1032-1038.

16. L. Smith, Polynomial invariants of finite groups, Res. Notes Math. 6, A.K. Peters Ltd., 1995.

17. E. Sperner, Ein Satz über Untermengen einer endlichen Menge, Math. Z. 27 (1928), 544-548.

18. R.P. Stanley, Weyl groups, the hard Lefschetz theorem and the Sperner property, Siam. J. Alg. Discr. Meth. 1 (1980), 168-184. 
19. R.P. Stanley, An introduction to hyperplane arrangements, Geom. Comb., IAS/Park City Math. 13, AMS, Providence, RI, 2007, 389-496.

20. W.A. Stein, et al., Sage mathematics software (Version 4.6.2), The Sage Development Team, 2011, http://wwwsagemath.org.

21. J. Watanabe, A remark on the Hessian of homogeneous polynomials, in The curves seminar at Queen's, Volume XIII, Queen's Papers Pure Appl. Math. 119 (2000), 171-178.

Department of Mathematics, Meijo University, Nagoya 468-8502, Japan

Email address: tmaeno@meijo-u.ac.jp

Department of Mathematical Sciences, Shinshu University, 3-1-1, Asahi, Matsumoto, Nagano 390-8621, JAPAn

Email address: nu@math.shinshu-u.ac.jp 